\title{
Outsider Influence And The Utility Of E-Mail As An Instrument For Teaching In Developing Nations: A Case Study In Fiji

\author{
Morris W. Shanahan, (Email: shanahanm@cpit.ac.nz), Christchurch Polytechnic Institute of Technology, New Zealand
}

\section{INTRODUCTION}

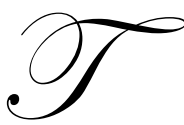

he impact of outsider influence in the advancement of human capital in developing nations is well documented ${ }^{1}$. This paper examines the utility of e-mail as a mechanism for delivery of outsider influence to middle managers in Fiji via a personal management development programme (PMDP). Thirteen participants took part in the PMDP over a six month period. The programme was aimed at enhancing their managerial skills by achievement of a series of negotiated objectives. There was one face-to-face meeting with each participant to set up the programme and negotiate objectives, and a second face-to-face meeting six weeks later to ensure all processes and systems were operational. During the six month duration of the programme, all other correspondence was limited to e-mail only.

At the end of the six month period a $360^{\circ}$ Assessment was conducted to determine achievement of objectives. Results showed that the process resulted in improvements of management skills in identified areas for all participants, supporting the contention that outsider influence can be successfully achieved using e-mail as a method for conveying information relevant to the teaching of management skills.

However, there were some issues germane to the use of e-mail in a developing nation that proved problematic. These issues related to: cultural constraints (i.e., issues of language), tradition-based limitations (i.e., paternalistic and hierarchical structures), an inherited past (British colonialism), and infrastructure (poor telecommunications services). All were obstacles that needed to be managed and sufficiently overcome, but once conquered, suggest web and internet based learning strategies can be successfully delivered at distance to individuals in developing nations.

"One of the challenges facing instructional designers is in producing e-learning systems, which take account of individual differences such as nationality, gender and more importantly from an educational perspective, cognitive learning style" (Graff, Davies \& McNorton, n.d.)

There are numerous arguments suggesting that the use of computer-mediated-communication (CMC) can overcome a number of constraints, both environmental and cultural (Bates, 1997; Garrison \& Anderson, 2003; Jin, 2005; Kanuka, 2005). Moran and Hawisher (1998) suggest that the speed of e-mail can assist in offsetting distance and time constraints. Jin (2005) argues that "web technology provides opportunities to bridge the geographical gap between the instructor and the learner" (p59). Jin also found that the use of e-mail as a teaching tool allowed distance learners a vehicle for rapid communication, "more time to reflect on their own ideas and improve critical thinking in their own pace and at a convenient time" (p64).

Sunderland (2002) found that e-mail was a useful tool in assisting PhD students at distance and she argues that the use of e-mail not only provides a convenient and fast method of communication, but also ads "personal and social value for its users" (p245). Young (2004) determined that the personalized attention afforded through e-mail was beneficial in assisting learners comprehend difficult issues and concepts. She also argues that online learning provides students with greater flexibility in relation to time and pace of learning, both of which are seen as beneficial.

\footnotetext{
${ }^{1}$ For more information on outsider influence in developing nations, see Shanahan and Shanahan, 2005.
} 
Jin (2005) found that students were more engaged when the learning was applied directly to relevant activities and online instruction is capable of being constructed to be precisely appropriate to each individual involved.

Bargh, McKenna, \& Fitzsimons (2002) argue that CMC may also provide individuals with the opportunity to be more open and honest as they feel freed from the social constraints inherent within physical interpersonal interactions, suggesting that the traditional barriers existing between teacher and learner may be reduced at distance. More frequent student-teacher interactions are afforded and advantageous.

But there are constraints in delivering learning at distance. Ragan and White (2001) suggest that the some of the strengths of e-mail communication (speed, volume) may also be weaknesses, as communication through writing only can generate an enhanced potential for miscommunication between participants. Halio (2004) agrees, suggesting that providing feedback can be difficult as the lack of visual interaction means feedback must be made clear and unambiguous, potentially more difficult given the shortened style of e-mail communication (Bertacco \& Deponte, 2005). Furthermore, Pate-Moulton, Klages, Erickson and Conforti Jnr (2004) assert, "writing is not every student's strongest ability" (p27). As one of their participants stated, "communicating with words on a computer screen seemed dry and lifeless" (p32). In essence, e-mail is one dimensional, limited to reading/writing only, and does not take into consideration the possibility that the student has a different individual learning style and/or preference.

Jin (2005) found motivational problems due to lack of any immediate monitoring systems could detract from the efficacy of e-mail as a learning tool and that frustrations due to technical difficulties could be problematic and impinge on participant learning. Halio (2004) discusses the gender differences in e-mail interaction which can pose some difficulties for effective teacher-participant interaction. She suggests that females may be more challenged in a distance learning environment and require more nurturing, encouraging correspondence, while the males are sensitive to criticism and can be more aggressive and assertive in their messages. It could be argued from the reduced-cues perspective that CMC may be an inappropriate modality for receiving personal feedback as ambiguity and miscommunication can be elevated.

When the constraints of culture and tradition, so strongly rooted in many developing nations (Banutu-Gomez, 2002), is textured in, the utility of e-mail as a learning tool can get further complicated. For example, numerous studies have identified cultural differences in learning styles (Bell, 1994; Park, 2000; Ramburuth, 2000; Reid, 1987). Pacific Island history and traditions are handed down orally (and visually via story-telling) from generation to generation (Hutakau, n.d.). These strong oral, visual and kinaesthetic tendencies are highlighted in the strong discrepancy between visual/oral and reading/writing skills in Pacific Island nations (Hutakau, n.d.). Such an incongruity could potentially render written e-mail communication even more problematic.

Learning success hinges on the Pacific Islander's attitude toward the teacher. Horsley (n.d.) states that in the Pacific "the teacher is seen as the one with all the power because of position and age" restricting free expression of contradictory or controversial views. Hutakau (n.d.) believes that only once high levels of trust have been developed between teacher and student do learners feel more confident of expressing opinions and ideas. Developing trust in a distance learning environment is challenging in that trust is traditionally based on face to face interactions, not ones developed without visual and/or oral interaction. Furthermore, "Polynesian students are not used to informal ways of learning, such as discussions and group work. They will be quite unwilling to express their own opinions because they have been brought up to believe all they need to know will be passed to them through the words of their teachers" (Horsley, n.d.).

Issues of language must also be contextualised as many developing nations have colonial pasts and language is mixed. Jin (2005) found some disadvantages in the use of e-mail as a communication vehicle, such as misinterpretations, are possibly due to issues of language and the loss of non-verbal communication. For example, Fiji has three primary languages; Fijian, Hindi, and English. Papua New Guinea has over 400 different indigenous languages, plus English and Tok Pigin are widely spoken. Such diversity of language makes a 'one size fits all' language approach difficult. Language utilisation is also different from that in many developed Western societies. For example, in the Pacific "The most distinctive feature of Pacific island culture is the concept that it is built on, the 
concept of "indirectness". Their way of life is like a form of 'social poetry' with imagery and metaphor everywhere" (Hutakau, n.d.). This makes the use of direct language rather complex irrespective of vernacular.

Another cultural difference is in the concept of time. In most developed nations, particularly Western ones, time is more of a mechanical construct, an instrument for regulation of activity. In the Pacific, time is a much more fluid, social construction "generalised in terms with the activity associated with it" (Hutakau, n.d.). Speed of communication afforded by e-mail and the internet is not necessarily a valid construct and therefore must proffer some issues in the use of e-mail as a learning device over space and time.

Lastly, in relation to developing nations, poverty poses a much more pointed ethical issue of technology access than in Western nations. While internet access is growing, most Pacific Island nations still lag well behind their Western counterparts in availability and affordability. In Fiji for instance, the environment is evolving and the traditional telco (Telecom Fiji) is now competing against private internet providers. But progress is slow. The provision of fast internet services to most businesses and their employees is rather restricted, both as a result of infrastructure and price constraints. For example, in Fiji internet supply is limited in most businesses to only a few terminals and access is strictly controlled due to the cost of the service. This is common practice in many companies in the South Pacific region.

Given that the majority of research supports the utility of CMC as a distance learning vehicle, results have primarily been limited to include only those countries already inculturated into the use of such new technologies. What about those emerging areas of the world where traditional, cultural and infrastructure constraints may render such methods more difficult to implement? Will CMC methods, such as the use of e-mail as a learning tool, be effective in these countries? This study examines the usefulness of e-mail as an educational instrument in a developing Pacific Island nation - the Republic of Fiji.

\section{METHOD}

Action research methods were utilised in the delivery of a Personal Management Development Programme (PMDP) to thirteen middle management executives in Fiji. Six were indigenous Fijians, five were Indo-Fijians, and two were of mixed Fijian/Indo-Fijian descent. Of the thirteen, five were female and eight were male. The PMDP is a management development programme which is delivered primarily via e-mail with two face-to-face visits; one to set up the process and gather information, and a second visit six weeks later to check that all systems and processes are operational $^{2}$. In this instance, the researcher was also the practitioner acting as 'management coach' for each participant in the PMDP.

All thirteen participants completed a Learning and Working Styles and a Lincoln/VARK questionnaire at the commencement of the programme to ascertain their preferred working and learning styles (see Appendix A). Results were exhibited in table form.

A record of all e-mail correspondence with each participant was maintained. Number of communications with each participant was examined to see if any differences existed between gender and/or race.

Finally, each participant was asked to complete a questionnaire at the end of the programme that related directly to e-mail as a delivery mechanism (see Appendix B). This questionnaire was sent to each person electronically within three months of completion of the PMDP. Results were categorised and presented in table form.

\section{LIMITATIONS}

While the practitioner-researcher method used in this study produced useful insights with respect to the effectiveness of e-mail as a delivery tool for a management development programme to participants in a developing nation, it has several limitations. First, this study does not represent a large, randomly-selected population and any

\footnotetext{
${ }^{2}$ For a complete explanation of the PMDP programme, see Shanahan and Shanahan (2005).
} 
extrapolation of results to other populations must be made with caution. Secondly, this study lacks a control mechanism making any comparatives impossible. Finally, the variables discussed in this study are situationally constrained, which highlights a distinction between empirical research and reflection as methods of scientific exploration.

\section{RESULTS}

There were no significant differences in working and learning styles, although Fijians tended to be slightly more holistic in approach than did Indo-Fijians (see Table 1).

Table 1: Number of participants by working and learning style

\begin{tabular}{ccccc}
\hline Working and Learning Style & Male & Female & Fijian & Indo-Fijian \\
Extremely Holistic & 0 & 1 & 1 & 0 \\
Somewhat Holistic & 2 & 2 & 2 & 1 \\
Both & 3 & 1 & 2 & 2 \\
Somewhat Analytical & 2 & 1 & 0 & 2 \\
Extremely Analytical & 1 & 0 & 1 & 0 \\
\hline
\end{tabular}

While results showed no significant differences between visual, oral or kinaesthetic as preferred or secondary learning styles, there was a difference between these three and reading/writing. None of the thirteen participants scored reading/writing as either their preferred or secondary learning style (see Table 2).

Table 2: Number of participants by preferred and secondary learning style

\begin{tabular}{ccc}
\hline Learning Style & Preferred style & Secondary style \\
Visual & 5 & 4 \\
Aural & 4 & 5 \\
Kinaesthetic & 4 & 4 \\
Reading/Writing & 0 & 0 \\
\hline
\end{tabular}

Of the thirteen questionnaires sent out concerning e-mail as a delivery mechanism, only seven were returned. Results were paraphrased, grouped together by similarity of response, and only those receiving more than one mention are exhibited in (see Table 3).

Table 3: Responses to questionnaire on the use of e-mail as a teaching/learning mechanism

\begin{tabular}{ll}
\hline Question & Responses \\
1. Overall impressions & Worked well (x5) \\
& Could get lost in the mix of other e-mails (x3) \\
& Was difficult at times when you were busy (x2) \\
& Got easier as time went by (x2) \\
& Immediacy of contact (x5) \\
2. Liked most & Knowing the 'coach' was only an e-mail away (x3) \\
& Deal with e-mails in your own time (x3) \\
& Distance made it easier to be honest (x3) \\
& Felt one-to-one (x2) \\
& Missed the non-verbal communication (x3) \\
& Using English exclusively (x3) \\
3. Disliked & The system going down (x3) \\
& Needed to be monitored more (x3) \\
& Easy to file and forget when busy (x2) \\
& Hard to always say what you feel in e-mails (x2) \\
& None (x4) \\
Interactive network for participants (x2) \\
It got better/easier as it progressed (x3) \\
5. Additional comments & Use video conferencing (x2) \\
\hline
\end{tabular}

The number and length of e-mails by gender and race was examined but no differences were found. 
Results indicate that issues of language and the lack of non-verbal communication appear to be somewhat problematic. While in Fiji English is the principle language of business, it is not the main language when it comes to interpersonal communication. As the PMDP relies on a close and trusting relationship between participant and provider, this result was not unexpected.

\section{DISCUSSION}

Overall, the use of e-mail as a delivery mechanism for the PMDP was successful with all thirteen participants completing the course and nearly all exhibiting significant improvement in their managerial/leadership skills (Shanahan \& Shanahan, 2005). But several issues had to be overcome by the practitioner-researcher and the participants to ensure success. These issues related to cultural differences and constraints; traditional structures; and matters of infrastructure. Each of these will be considered in context of the results above and practitioner-researcher observations.

\section{Cultural Differences And Constraints}

The results of this study uncovered several cultural differences between the Western practitioner-researcher and the participants that were problematic, as well as several constraints between the indigenous Fijian cultures that needed to be addressed. It was apparent that issues of language, both vernacular and cultural utilisation, posed significant constraints on the utility of e-mail as an educational delivery mechanism. The use of English as the sole communication language was one issue, but the actual constraints inherent within e-mail communication was another, as was the differences in learning styles apparent between differing ethnicities. While these constraints tended to be at least partially subdued over the duration of the PMDP, there were even issues towards the conclusion of the programme that are worthy of mention. As the relationship between practitioner-researcher and participant intensified and became more personal and trusting towards the conclusion of the programme, one participant commented "I found it hard to express what I really meant later on in the programme because I couldn't find the right words [in English]." Another commented that it was difficult to "write down my thoughts and feelings in English" and would have preferred to use her own language. While the overall result was one of achievement, even in Fiji where English is widely accepted and spoken, lack of fluency in the indigenous languages by the practitionerresearcher was a constraining factor.

E-mail delivery was also problematic in that it is written and fails to engage the predominant learning styles of participants, namely visual, aural and kinaesthetic. Results of the Lincoln VARK Test showed that none of the participants exhibited either a first or second preference for reading/writing as a learning style, contrary to tests conducted in the West which exhibit some degree of preference for this learning modality. Yet e-mail is virtually constrained to this mode of information transfer. As suggested in Understanding the differences (n.d.), it is best to use learning strategies that are congruent with the particular learner's preferred learning style. Therefore delivery of the PMDP almost exclusively by e-mail was, at times, clumsy. The practitioner-researcher quickly realised through feedback from participants that a less Westernised written style of presentation of material was required. They were not used to the more formulaic, stylised, mass presentation tended to be used in the West and wanted more explanation and clarification as to the personal relevance and consequence of the information. As the practitionerresearcher learned over time, presenting material in more pictorial form and weaving sometimes lengthy narrative into the e-mails was effective. These narratives were often related directly to the participant's environment, personality and/or family/work situation. One participant subsequently commented "Now I 'see' what you mean", while another replied to a clarifying e-mail "now I 'hear' where you are coming from". This was a significant breakthrough in the delivery of the PMDP and progress after this discovery was much more rapid and e-mail communication increased between the practitioner-researcher and the participants.

Providing participants with more active tasks was also useful, lending support to Pate-Moulton et. al.(2004) and Hutakau (n.d.). As one participant commented "It [the PMDP] was much better when you gave me things that I [physically] had to do." Another stated "I learned more when you sent me stories about real people, especially other Fijians that I could relate to and learn from", rather than more directed readings. Dufficy (n.d.) was correct in arguing that in the Pacific Islands, flexible teaching methods and useful, relevant activities tend to work best. 
When utilising e-mail as a feedback mechanism, the practitioner-researcher agrees with Halio (2004) that great care needs to be taken in the wording of any evaluation. As Bertacco and Deponte (2005) found, personal email correspondence tends to be shorter and perhaps less emotional and/or personal than similar correspondence conducted through letter writing. As Hutakau (n.d.) suggests, Pacific Island people are far more sensitive than they may appear on the surface. This resulted in one delicate situation. Feedback to one participant included a glowing report that included one brief, couched reference to a comment about his behaviour being too "one of the boys" in nature. The participant took this comment completely out of context and was very upset by it, so much so that it took numerous e-mails and even a telephone call to get him to recognise the lack of severity of a minor criticism and to relish the overall positive nature of the report. It was immediately apparent that the cultural necessity in Fiji of presenting an outward appearance devoid of emotion was not what was actually taking place internally, and the need to be responsive to particular cultural sensitivities in all e-mail contact became glaringly apparent. This continued to be somewhat of a challenge when using e-mail due primarily to the abbreviated nature of the correspondence, confirming Ragan and White's (2001) arguments that written e-mail communication affords greater opportunities for miscommunication. While the practitioner-researcher modified the length and nature of feedback e-mails to make them more personal and less formal, even at the end of six months there appeared room for further improvement. The results of this study suggest that before attempting e-mail delivery of such a programme, the provider must be fully aware of the cultural sensitivities and nuances before embarkation and modify any Westernised e-mail style to take such differences into account.

Another cultural difference was a source of some tension for the practitioner-researcher, particularly in the early days of delivery of the PMDP. That issue related to the concept of time. Westerners tend to view time as a segmented structure that is concrete and fixed. In Fijian culture time is more fluid and only relevant to the activity at hand. As a result, imposition of deadlines for e-mail correspondence became problematic for the practitionerresearcher and one that took some time for him to overcome. Adherence to time constraints is simply not a cultural commitment and imposition of such deadlines is contrary to the thinking of indigenous Fijians in particular. The result was some anxiety on behalf of the practitioner-researcher that had to be resolved appropriately. This was accomplished by setting 'parameters' rather than absolute deadlines for correspondence, and frequent e-mail reminders to participants was also required. This appeared to result in increased compliance as the programme progressed, although any linkages would be tenuous.

Despite the cultural constraints, over the course of the PMDP the utility of e-mail as a method of communication became appreciated and seemingly more effective. As one participant stated "In the last few weeks of the course I started to really look forward to your e-mails as they painted really clear pictures of what I needed to do." The results of this study highlight the importance of provider knowledge and understanding of the cultural communication differences, particularly language issues, into which any distance programme is to be delivered, as well as a willingness to modify delivery to meet the needs of learners in developing nations.

\section{Traditional Structures}

This study found support for Jin's (2005) claim that for learners to take advantage of e-mail-based training programmes, participants with exposure to traditional, highly directed learning environments must adjust their perceptions and attitudes to learning and adopt a more student-driven approach. The practitioner-researcher experiences in this study confirm Jin's claim. This was partially overcome by negotiating agreements and gradually placing more of the onus for learning on the participant. For most this was a new experience and several relished the opportunity. One stated "I loved organising my own learning. It was really exciting and I had never done anything like that before." However, one other believed it was all too hard as "I just couldn't motivate myself. You weren't here so I didn't feel like I had to do it [tasks and readings]." Researcher observation suggests that over half made some relatively robust attempts at self-directed learning, while the remainder made varying levels of effort. What was apparent with all participants was that this was the first time they had actually been presented with such an opportunity.

Part of the lack of self-direction is a result of the Power Distance (Hofstede, 1980, 1984) that exists in many developing nations. In both Fijian and Indo-Fijian cultures this Power Distance fosters a hierarchical, paternalistic 
environment where learners follow rather than lead, seldom react to or challenge decisions and/or existing structures, and rarely express their own views or opinions (Schuler and Rogovsky, 1998). This is further exacerbated by the collective nature of Fijian society across both major ethnicities. In the Pacific, cultural and social rigidity constrains individual expression rather than freeing it up (Hautkau, n.d.). The Power Distance constraint was a source of tension at several levels.

Traditional, colonial structures (Consedine, 2004) are still prominent in Fiji, particularly in education where the teacher is the holder of power. This can be problematic when using e-mail as a primary delivery mechanism as participants in this research initially considered themselves in a teacher-student relationship and sought a highly directed approach. The practitioner-researcher broke these barriers by using e-mail correspondence to develop trust with participants. A far less formal approach to e-mail communication was introduced with longer, more frequent emails that strived at building a trusting rapport between the practitioner-researcher and the participant and placed them on a more equal footing. Non-relevant but personally revealing matters were discussed, such as family, sport and food, to generate a more open feeling of equality. This appeared to succeed as participant e-mails became generally more revealing and increased in length, confirming the arguments of Bargh, McKenna, \& Fitzsimons (2002).

Findings also tended to confirm Young's (2004) argument that more personalised use of e-mail is beneficial for learners. This study found that the personalisation of the process was useful but is time consuming. Each e-mail communication had to be individually crafted making any economies of scale redundant. In essence, each participant ended up with an individualised learning programme, rendering any efficiencies in using CMC as a delivery mechanism unproductive. This runs somewhat contrary to the arguments that suggest CMC can be an efficient method for mass educational delivery.

A second, unforeseen issue relating to the structural constraints inherent within Fijian society was the inability to use internet chat rooms or web-based programmes to encourage a collective learning environment. The paternalistic, hierarchical constraints makes programmes such as Blackboard problematic in that group interaction cannot be, due to the nature of society, a collegial concern. Such programmes adopt a more Westernised, egalitarian approach that simply does not recognise the traditional structures that exist in a country like Fiji. It could only be useful if all participants were of the same level within society. For example, this study one of the participants in the PMDP programme was an indigenous Fijian male of Chiefly stature. It would have been culturally inappropriate for the less senior participants, particularly the female participants, to interact with him via the internet, and virtually impossible for anyone to question or challenge his ideas on any particular topic. In essence, use of such a tool would have been de-motivating and dampened any possible learning opportunities. Other issues, such as gender, religious orientation and age could also pose barriers to the efficacy of such web-based learning tools. As a result, all e-mail activity was maintained solely between the practitioner-researcher and each individual participant. This requires more input from the provider than would be expected in more collegial, Western environments.

A serendipitous finding suggests that Bargh, McKenna, \& Fitzsimons (2002) were correct in their argument that participants feel less constrained by social and traditional constraints when using e-mail as the primary source of communication, but this was limited to practitioner-researcher-participant interactions. Indeed, the practitionerresearcher made note of several instances where individual participants became more open and emotive as they gained confidence in the practitioner-researcher and e-mail as a communication medium. While it is difficult to tease these variables apart, one participant did comment that "using e-mail and knowing you [the practitioner] were far away in New Zealand seemed to make it easier for me to tell you things I wouldn't talk about with anyone else". This was mirrored by another participant comment "It was easier to talk to you on e-mail as I could be more honest than if we were face to face." It could be that the use of e-mail as a learning tool could reduce the traditional Power Distance constraints and enhance learning. This is certainly an area in need of further investigation.

Overall, the constraints of tradition on the use of e-mail as an educational tool in Fiji were overcome. However, these limitations do pose some problems for outside providers considering implementation of learning programmes into developing nations using CMC. In this instance, the collegiality afforded by internet and web-based programmes would detract from learning, not enhance it. Furthermore, any provider would have to reconsider the teacher-learner relationship in light of varying traditional structures and expectations. But this study does show that 
these constraints are not insurmountable and can be either overcome or detoured provided the outside deliverer is cognizant of the issues and prepared to work through the limitations.

\section{Infrastructure Issues}

Fiji boasts one of the higher standards of living in the South Pacific Island region, yet even here there were financial and infrastructure constraints on the use of e-mail as a proper delivery mechanism for the PMDP. Two issues are of particular note: lack of access to the internet, and poor quality provision of services.

According to Robertson and Sutherland (2001) close to 80 per cent of the indigenous Fijian population are economically disadvantaged, while the figure nears 90 per cent for the Indo-Fijian population. The result is that computer penetration into households is very limited and access to terminals at work is well below that of most developed countries. This certainly has a restrictive impact on the use of $\mathrm{CMC}$ as an educational apparatus. Of those involved in the PMDP, only one had internet access at home, while just over half had internet access at their workstation. The others were required to access their e-mail at public terminals either at work or at public internet providers, such as e-cafes. As much of the material in the PMDP was of a relatively personal nature, this proved awkward for some participants. One commented that she was "highly embarrassed when I found a colleague reading one of the e-mails I had printed out" even though there was no particular private information in the e-mail. The printer was simply in a different area to the access terminal. Another commented that she waited until "everyone had gone for lunch or gone home" before she opened her e-mails. She felt she had the right to privacy, but as her only access was a shared terminal in the middle of an open plan office, privacy had to be manipulated rather than considered a right as it might in Western societies.

The other infrastructural constraint was the inconsistency of internet service by the sole provider of internet in Fiji - Telecom Fiji. The internet was slow, fraught with interruptions and often 'down' for considerable periods of time. The researcher's experience suggests this is the situation across most Pacific Island nations from Samoa to Papua New Guinea to the Solomon Islands. While very few e-mails were actually 'lost', the lack of a consistent service was, to quote one participant, "extremely frustrating! Just when you had time to get to your PMDP stuff, the net would go down." This sentiment was more or less expressed by several other participants. Again, overcoming this constraint required patience and the practitioner-researcher re-evaluating his concept of time as a rigidity. By setting parameters for participant responses, the difficulties presented by lack of service were overcome.

\section{CONCLUSION}

Mariola and Manley (2002) propose that distance learning can be effective provided certain criteria are met. These include: a sufficient level of comfort with this form of delivery by both participants and deliverers; adequate technical support; frequent communication and monitoring; and detailed presentation of topics and instructions. However, for distance learning via e-mail to be effective in developing nations, such as Fiji, there are additional constraints that need to be attend to if success is to be achieved. These include the cultural, traditional and infrastructure constraints inherent within the society, as well as any pre-conceptions held by the provider.

As Dufficy (n.d.) argues, to teach in the South Pacific, providers must recognise their own particular cultural expectations, garner high levels of trust, be flexible, maintain relevancy, understand traditional constraints, and get to know the learner and their wider family. Once these criteria are met, learning can be optimised. The results of this study confirm Dufficy's views. The use of e-mail as a teaching and learning method was useful, but only once the practitioner-researcher was able to understand and overcome the constraints of delivery. This is a good lesson for any outsider considering using the internet as an educational delivery mechanism to developing nations. 


\section{REFERENCES}

1. Banutu-Gomez, M.B. (2002). Leading and managing in developing countries: Challenge, growth and opportunities for twenty-first century organisations. Cross Cultural Management, 9(4), 29-42.

2. $\quad$ Bates, T. (1997). The future of educational technology. Learning Quarterly, 1 (2), 7-16.

3. Bargh, J. A., McKenna, K. Y. A., \& Fitzsimons, G. J. (2002). Can you see the real me? The activation and expression of the 'true self' on the Internet. Journal of Social Issues, 58,(1), 33-48.

4. Bell, Y. R. (1994). A culturally sensitive analysis of black learning style. Journal of Black Psychology, 20,(1), 47-61.

5. Bertacco, M. and Deponte, A. (2005). E-mail as a speed-facilitating device: A contribution to the reducedcues perspective on communication. Journal of Computer-Mediated Communication, 10(3), article 2. http://jcmc.indiana.edu/vol10/issue3/bertacco.html

6. Consedine, R. (2004). Treaty of Waitangi Awareness Workshop, 30 April, 2004, CPIT, Christchurch, New Zealand.

7. Dufficy, P. (n.d.). The educators views 1: Paul Dufficy. Retrieved online 20 October 2005 at http://alex.edfac.usyd.edu.au/AcrossCurric/Paradise/M.H's\%20\% 20ParaProb.html\#INDEX

8. Garrison, D. R. \& Anderson, T. (2003). E-Learning in the 21 st Century: A Framework for Research and Practice. London: Routledge/Falmer.

9. Graff, M., Davies, J., and McNorton, M. (n.d.). Cognitive style and cross cultural differences in Internet use and computer attitudes. Retrieved online 20 October 2005 at http://www.eurodl.org/materials/contrib/2004/ Graff_Davies_McNorton.html).

10. Halio, M. P. (2004). Teaching in our pajamas: negotiation with adult learners in online distance writing courses. College teaching, 52,(2), 58-62.

11. Hofstede, G. (1980). Motivation, leadership, and organisation: Do American theories apply abroad? In D. W. Organ, (Ed.), The applied psychology of work behaviour. Boston: Irwin.

12. Hofstede, G. (1984). Culture's consequences: International differences in work-related values. London: Sage.

13. Horsley, M. (n.d.). Educator views 3: the Australian education system and Polynesian students interview with Mike Horsley. Retrieved online 20 October 2005 at http://alex.edfac.usyd.edu.au/AcrossCurric/Paradise/M.H's\%20\% 20ParaProb.html\#INDEX

14. Hutakau, I. F. (n.d.). A multicultural approach to learning: Pacific Island students. Retrieved online 20 October 2005 at http://alex.edfac.usyd.edu.au/ AcrossCurric/Paradise/ M.H's\%20\%20ParaProb.html\#a\%20concept\%20of \%20time.

15. Jin, S. H. (2005). Analyzing student-student and student-instructor interaction through multiple communication tools in web-based learning. International Journal of Instructional Media, 32(1), 59-67.

16. Kanuka, H. (2005). An exploration into facilitating higher levels of learning in a text-based internet learning environment using diverse instructional strategies. Journal of Computer-Mediated Communication, 10(3), article 8. http://jcmc.indiana.edu/vol10/issue3/kanuka.html

17. Mariola, E. and Manley, J. (2002). Teaching finance concepts in a distance learning environment - a personal note. Journal of Education for Business, 77,(3), 2002, 177-180.

18. Moran, C. and Hawisher, G. (1998). The rhetorics and language of electronic mail. In Snyder (Ed.) Page to screen. Taking literacy into the electronic era. London: Routledge.

19. Park, C. C. (2000). Learning style preferences of southeast Asian students. Urban Education, 35,(3), 245-268.

20. Pate-Moulton, S., Klages, C., Erickson, A., and Conforti Jnr., P. A. (2004). Integrating technology and pedagogy: the DLP (Distance Learning Partners) project investigates literature circles. Curriculum and teaching dialogue, 6,(1), 25-34.

21. Ragan, T. J. \& White, P. R. (2001). What we have here is a failure to communicate: The criticality of writing in online instruction, Computers and Composition, vol 18, pp. 399-409.

22. Ramburuth, P. (2000). Cross cultural learning behaviour in higher education: perception versus practice. Paper presented to the Seventh International Literacy and Education Research Network (LERN) Conference on Learning, RMIT University, Melbourne, 5-9 July 2000.

23. Reid, J. (1987). The learning style preferences of ESL students. TESOL Quarterly, 21,(1), 87-111. 
24. Robertson, R and W Sutherland 2001, Government by the Gun: The Unfinished Business of Fiji's 2000 Coup, Pluto Press, Sydney.

25. Schuler, R.S. and Rogovsky, N. (1998). Understanding compensation practice variations across firms: The impact of national culture. Journal of Business Studies, 29(1), 159-178.

26. Shanahan, M.W. and Shanahan, Y.P. (2005). The development of human capital in emerging nations: The utility of Personal Management Development Plans at Communications Fiji Limited. In P. Hutchison (ed.) Proceedings of the $18^{\text {th }}$ Annual SEAANZ Conference, Armidale, Australia. University of New England Publications CD-ROM, ISBN: 186389957 X. p. 1-18.

27. Sunderland, J. (2002). New communication practices, identity and the psychological gap: the affective function of e-mail on a distance doctoral programme. Studies in Higher Education, 27(2), 233-246.

28. Understanding the results. (n.d.). Retrieved online 25 November 2006 at http://www.varklearn.com/english/page.asp? $\mathrm{p}=$ understandingresults.

29. Young, J. (2004). Virtual schools: personalizing learning online. Media \& methods, 41,(2), 11-13.

APPENDIX A

Thinking and Working Styles Questionnaire

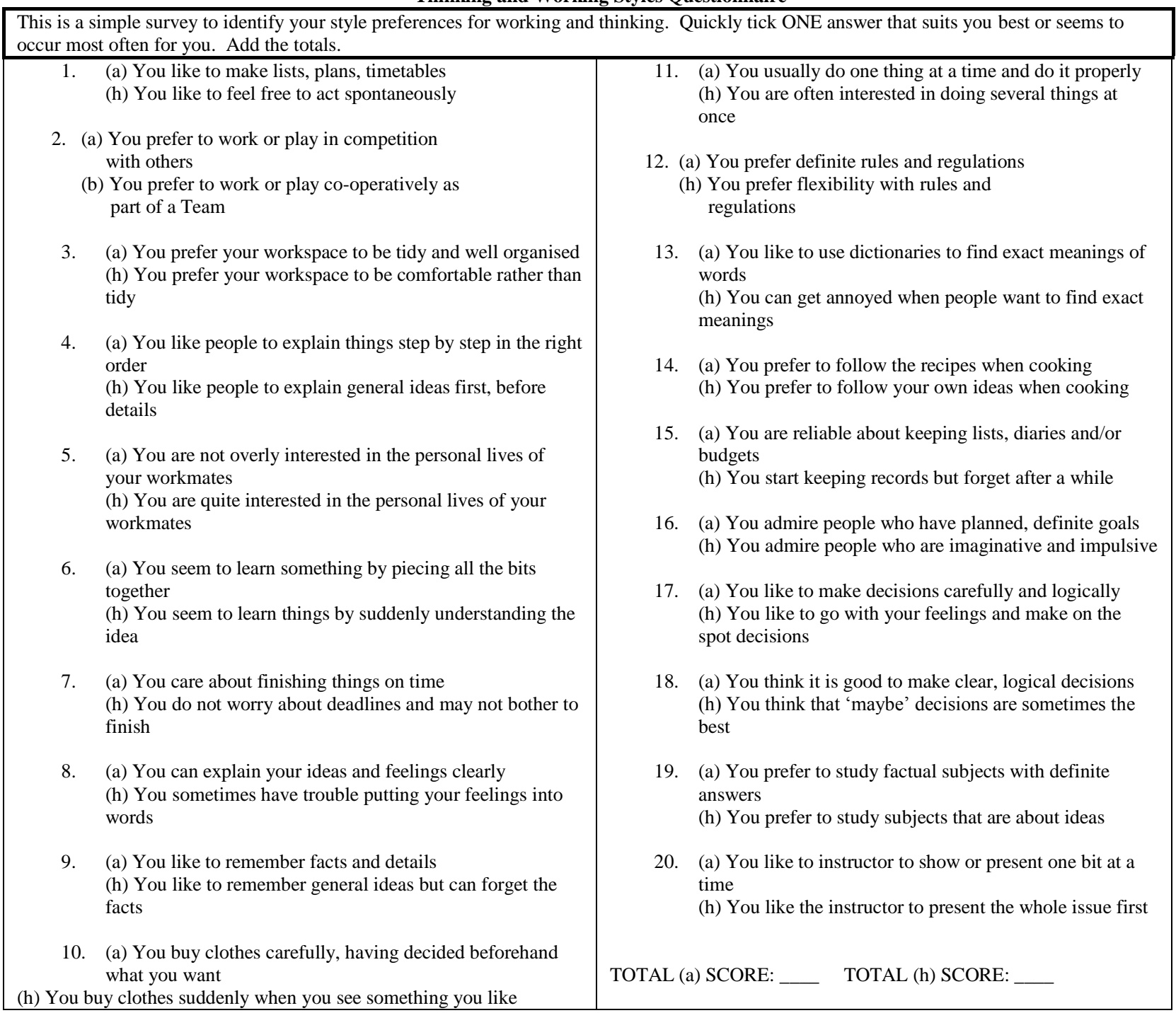


LINCOLN VARK Questionnaire: This is a quick survey to indicate which senses you prefer to use for learning. Quickly tick the answers which suit you most often in each case. Add the totals.

1. To relax you prefer to:

(V) watch TV/videos/DVDs

(A) talk to people or listen to something

(R) read something

(K) be active or play sport

2. When giving people directions, you would:

(A) tell them what to do

(R) write the directions down for them

(V) show them on a map

(K) use gestures and actions

3. You are most likely to get distracted by:

(K) people or things moving around

(V) the way something looks

(R) words used or spelled incorrectly

(A) noises

4. You solve problems most easily by: (R) writing details

(A) talking through the possibilities

(K) hands on practical experience

(V) sketching out possible solutions

5. While waiting (e.g. in a queue) you would:

(K) wriggle, fidget, move your feet/hands

(V) watch people or the scenery

(A) talk to yourself or others

(R) read or write something

6. To show sympathy you would most likely:

(R) write a letter

(V) choose a nice card to send

(A) telephone the person

(K) visit the person if possible
7. You like your projects to:

(V) look right

(A) sound right

(K) feel right

(R) use words correctly

8. In class you prefer:

(A) lectures and discussions

(K) experiments and activities

(R) taking notes or reading them

(V) diagrams, pictures, videos

9. You would be more likely to ask: (K) do you get the idea?

(V) can you see what I mean?

(A) do you hear what I'm saying?

(R) will you make a note of this?

10. To learn some poetry you would:

(R) read it over many times

(K) move around to get the rhythm

(A) say it out loud

(V) imagine the ideas visually

11. You judge people's moods by;

(V) looking at their faces

$(\mathrm{R})$ reading their memos

(A) listening to their voices

(K) noticing their gestures

12. You prefer the humour of:

(A) comedians who talk a lot

(K) action comedy

(R) literary wit

(V) colourful comics and cartoons

13. When buying clothes you mainly:

(R) study the labels

(K) feel the fabrics

(V) enjoy the colours/designs

(A) talk about the possibilities with someone
14. When visiting friends/family, you like to:

(K) be involved in physical activities

(R) read or play word games

(V) watch others do things

(A) discuss things

15. You prefer something explained by:

(V) diagrams, pictures, maps

(A) talk, lecture. discussion

(R) written instructions

(K) practical demonstrations

16. To tell friends about a holiday, you would:

(A) phone them to tell them

(R) write a letter or send an e-mail

(V) show them your pictures

(K) visit them to share your experiences

17. You can listen to a speaker best if you can:

(R) take your own notes

(K) move around (or the speaker does)

(A) close your eyes (or look away)

(V) see the speaker clearly

18. What you remember most about people is:

(A) things they have said

(K) things they have done

(R) things they have written

(V) how they look

TOTALS:

$\mathrm{V}=$

$\mathrm{A}=$

$\mathrm{R}=$

$\mathrm{K}=$ 


\section{APPENDIX B}

Date

Dear (participant)

What follows are a few questions concerning the use of e-mail as a delivery mechanism for the PMDP. As a graduate of the programme, I hope that you wouldn't mind answering four short questions. Your responses will be completely confidential and will perform two important tasks as the results will be used: (1) as part of an analysis of the usefulness of e-mail as a delivery mechanism, and (2) to modify delivery of the PMDP programme to further enhance its effectiveness. If you are happy to take part, please simply reply to this e-mail including your answers to the following questions. Your assistance is greatly appreciated.

Question One: What are your overall impressions of using e-mail as the primary delivery tool for the PMDP? (Please give some detail to your responses.)

Question Two: What did you like most (if anything) about using e-mail as a means of communicating during the PMDP? Explain.

Question Three: What did you like least (if anything) about using e-mail as a means of communicating during the PMDP? Explain.

Question Four: If you could change one or two things about the way the course was delivered to make it a better programme, particularly in relation to the use of e-mail, what would you do? (Please give some detail to your responses.)

Question Five: Are there any other comments you would like to make about the use of e-mail as the delivery method for the PMDP?

Thank you very much for your responses. They will be utilised to benefit future participants. If you have any questions, please contact me for clarification either via e-mail or telephone.

Morris W Shanahan

shanahanm@cpit.ac.nz

+6439408431 